\title{
Tabularia
}

\section{Comment Roger de Barneville est-il mort ? Sur les rapports entre les chroniques latines de la première croisade}

How did Roger of Barneville die? About Relationships among the Latin

Chronicles on the first Crusade

Com'è morto Ruggero di Barneville? Sui rapporti tra le cronache latine sulla prima Crociata

\section{Edoardo D'Angelo}

\section{(2) OpenEdition}

Journals

Édition électronique

URL : http://journals.openedition.org/tabularia/2880

DOI : $10.4000 /$ tabularia. 2880

ISSN : 1630-7364

Éditeur :

CRAHAM - Centre Michel de Boüard, Presses universitaires de Caen

Référence électronique

Edoardo D'Angelo, «Comment Roger de Barneville est-il mort ? Sur les rapports entre les chroniques latines de la première croisade », Tabularia [En ligne], Mémoires normandes d'Italie et d'Orient, mis en ligne le 19 juin 2017, consulté le 20 avril 2019. URL : http://journals.openedition.org/tabularia/2880 ; DOI : 10.4000/tabularia.2880 



\title{
Comment Roger de Barneville est-il mort? Sur les rapports entre les chroniques latines de la première croisade
}

\section{How did Roger of Barneville die? About Relationships among the Latin Chronicles on the first Crusade \\ Com'è morto Ruggero di Barneville? Sui rapporti tra le cronache latine sulla prima Crociata}

\author{
Edoardo D'ANGELO \\ Università degli Studi Suor Orsola Benincasa - Napoli \\ edoardo.dangelo@unisob.na.it
}

\begin{abstract}
Résumé :
L'article propose une analyse des rapports «génétiques» entre les chroniques latines de la première croisade, et porte une attention particulière à l'Hystoria de via et recuperatione Antiochiae atque Ierusolymarum, à travers la confrontation des différents récits de la mort du croisé normand Roger de Barneville. L'étude de cet épisode et d'autres sources des croisades conduit à penser que les textes connus comme les Gesta Francorum et l'histoire de Pierre Tudebode avaient pour modèle un texte aujourd'hui perdu.
\end{abstract}

Mots-clés : croisade, chroniques, Gesta Francorum, Hystoria de via et recuperatione Antiochiae atque Ierusolymarum

\begin{abstract}
:
Studies of the relationships among the Latin chronicles on the first Crusade, particularly looking to the Hystoria de via et recuperatione Antiochiae atque Ierusolymarum, analysing the different accounts of Roger of Barneville's death fighting against Turks. The analysis of this episode demonstrates that the so called Gesta Francorum and Petrus Tudebodus derive from an original account, now lost.
\end{abstract}

Keywords: Crusade, chronicles, Gesta Francorum, Hystoria de via et recuperatione Antiochiae atque Ierusolymarum

\section{Riassunto:}

Analisi dei rapporti "genetici» tra le cronache latine sulla I Crociata, con particolare attenzione alla Hystoria de via et recuperatione Antiochiae atque Ierusolymarum, mediante il confronto del racconto della morte del crociato normanno Ruggero di Barneville. L'analisi di quest'episodio nella Historia $e$ in altre fonti crociate conduce a pensare che $i$ testi not $i$ come Gesta Francorum e Pietro Tudebodo avessero alle spalle un altro testo originario, attualmente perduto.

Parole chiave: Crociata, cronache, Gesta Francorum, Hystoria de via et recuperatione Antiochiae atque Ierusolymarum

Tabularia «Études», «Mémoires normandes d'Italie et d'Orient», 2017, p.1-14, 19 juin 2017 URL : http://tabularia.revues.org/2880 | DOI : 10.400o/tabularia.2880 
Les sources latines de la première croisade dont nous figurons la liste ci-dessous sont nombreuses et variées, et présentent des problématiques et des positions idéologiques différentes, notamment parce que la première croisade ne fut pas une expédition «monolithique », mais rassembla des troupes de maintes régions de la France auxquelles se sont joints aussi les Normands de l'Italie méridionale.

\begin{tabular}{|l|l|l|}
\hline Gilles de Paris & Historia Vie Hierosolimitane & AeP \\
\hline Albert d'Aix & Historia Ierosolimitana & AA \\
\hline Baudri de Dol & Historia Jerosolimitana & BD \\
\hline Anonyme & Chronica monasterii Casinensis & chCas \\
\hline Foucher de Chartres & Historia Hierosolymitana & FC \\
\hline Anonyme & $\begin{array}{l}\text { Gesta Francorum et aliorum } \\
\text { Hierosolimitanorum (Histoire anonyme..., } \\
\text { éd. L. Bréhier, 1924) }\end{array}$ & GF \\
\hline Guibert de Nogent & Dei Gesta per Francos & GN \\
\hline Anonyme & $\begin{array}{l}\text { Hystoria de via et recuperatione Antiochiae } \\
\text { atque Ierusolymarum (olim Tudebodus } \\
\text { imitatus et continuatus) }\end{array}$ & HAI \\
\hline Pierre Tudebode & Historia de Hierosolymitano itinere & PT \\
\hline Raimond d'Aguilers & Le «Liber » de Raymond d'Aguilers & RA \\
\hline Raoul de Caen & $\begin{array}{l}\text { Gesta Tancredi in expeditione Hierosalymi- } \\
\text { tana }\end{array}$ & RC \\
\hline Robert le Moine & Historia Iherosolimitana & RM \\
\hline
\end{tabular}

Les auteurs de ces textes, comme en témoignent leurs noms, viennent euxmêmes de divers horizons (Provençaux, Normands de Normandie, Anglais, Lorrains, Normands d'Italie, etc.), mais certains sont restés anonymes.

Dans le présent article, nous traiterons des rapports génétiques réciproques entre ces textes, c'est-à-dire les relations textuelles, philologiques et internes au contenu. Il s'agit en vérité d'un sujet qui a déjà suscité de nombreuses études, mais elles se sont intéressées presque exclusivement au contenu des textes, et peu à la forme, c'est-à-dire aux faits philologiques (ou questions d'ecdotique) et au style ${ }^{1}$.

\section{Rapports génétiques entre les sources de la première croisade :} état de la question

Le premier problème concerne un groupe de textes, qui semblent dériver d'une même source : les Gesta Francorum (GF) ${ }^{2}$. John France dessine une carte dans

1. L'article s'inspire de la recherche publiée dans HAI, éd. D'ANGELO, 2009, «Introduzione ».

2. KreY, 1928, p. 57-78; Russo, 2001, p. 51-66; WOLF, 1991, p. 207-216. 
laquelle les GF sont pratiquement au centre de tous ces textes. Mais ce stemma est peu fiable pour plusieurs raisons ${ }^{3}$. Les problèmes viennent surtout de la position de l'œuvre de Raoul de Caen (RC) et de celle de la Chronique du Mont Cassin (chCas), qui n'ont aucune dépendance avec d'autres textes. Hartmut Hoffmann, le savant éditeur de la chCas, commet une erreur en considérant que le chapitre 4.11 dérive d'un mélange GF + RA + HAI et dit qu'aucun des trois textes ne peut être à lui seul tenu pour le modèle de la chronique ${ }^{4}$. En effet, la chCas est antérieure à 1115 tandis que l'HAI a sûrement été composée entre 1130 et 1153 .

Il est établi que de GF dérivent probablement BD, GN, PT, RM, HAI. Mais les GF coïncident à $80 \%$ avec le texte de Pierre Tudebode (PT). Le tableau suivant donne les hypothèses critiques, proposées par les éditeurs des chroniques (dont les noms figurent dans la première colonne), sur les liens entre les GF, la chronique dite de Pierre Tudebode, et les autres textes qui lui sont liés :

\begin{tabular}{|l|l|l|}
\hline $\begin{array}{l}\text { von Sybel, Thurot, } \\
\text { Bréhier, France }\end{array}$ & GF $\rightarrow$ PT & \\
\hline (Bongars), RHC & $\begin{array}{l}\text { PT } \rightarrow \text { GF }(\text { GF }=\text { Tudebodus } \\
\text { abbreviatus })\end{array}$ & \\
\hline Hagenmeyer & GF $\rightarrow$ RA & RA $\rightarrow$ FC \\
\hline Klein, Iorga & RA $\rightarrow$ GF & \\
\hline Hill, Hill & $\begin{array}{l}\text { source commune } \rightarrow \text { GF, PT, } \\
\text { RA }\end{array}$ & \\
\hline Rubenstein & Jerusalem History (GF + PT) & \\
\hline Flori & texte en évolution & (Hill + Rubenstein) \\
\hline
\end{tabular}

Venons-en maintenant à l'Historia Antiochiae atque Ierusalem (HAI). Ce texte apparait presque entièrement comme une compilation de sources $(\mathrm{GF}+\mathrm{PT}+\mathrm{RA}+\mathrm{RC})$. Il est contenu dans le ms 300 du Mont Cassin daté du $\mathrm{XIII}^{\mathrm{e}}$ siècle. Pour compliquer la situation, il se trouve que l'HAI est, elle-même, la source d'une autre compilation de sources sur la croisade (GF + PT + RA + HAI notamment), compilation qui est contenue dans le ms Paris, BnF lat. $6041 \mathrm{~A}$ daté $\mathrm{du} \mathrm{XIV}^{\mathrm{e}}$ siècle. Cette situation crée une série de problèmes importants sur le plan théorique de l'édition critique du texte : quelle valeur stemmatique faut-il donner au témoin (de tradition indirecte, naturellement) parisien ? L'auteur anonyme de l'HAI compile, nous l'avons dit, quatre sources : GF + PT + RA + RC, pour un total de $80,3 \%$ du texte; les $19,7 \%$ restant pourraient être des parties «originales» :

3. France, 1998, p. 29-42, en part. p. 42.

4. «Keine der drei Texte kann für sich allein die Vorlage der Chronik gewesen sein », chCas, éd. Hoffmann, 1980, p. xxix. 


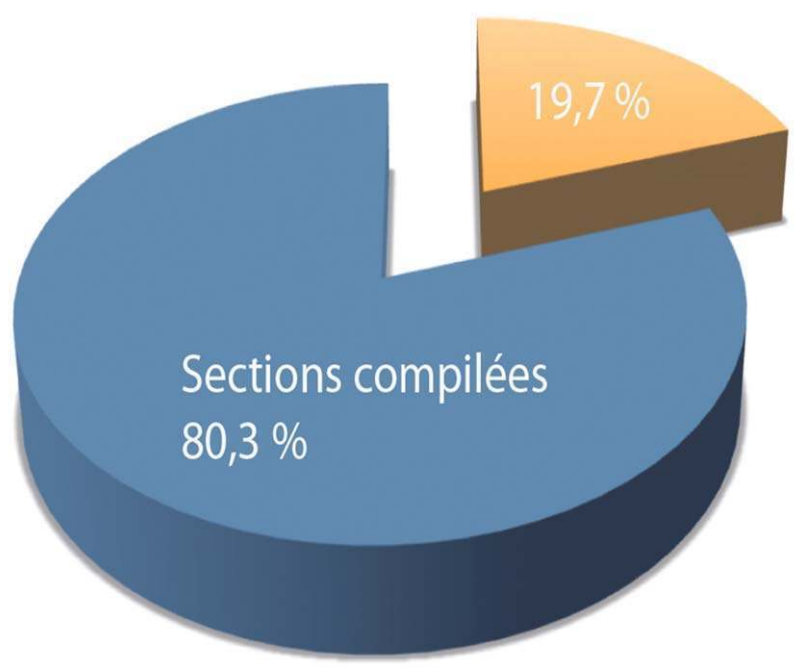

Fig. 1 : Parties compilées et parties originales de l'HAI

L'anonyme de l'HAI compile sa chronique en utilisant des stratégies différentes; selon les cas, soit il copie littéralement ou modifie très peu, soit il réécrit entièrement le latin difficile et très rhétorique de Raoul de Caen (RC), en résumant et en simplifiant le texte complexe de l'écrivain normand.

En ce qui concerne les sections originales (19,7\%), elles présentent ellesmêmes une difficulté : sont-elles une création autonome (réellement originale) de l'anonyme, ou ne remontent-elles pas plutôt à quelque source aujourd'hui perdue ? Donner une réponse à cette question peut probablement conduire à éclairer d'une lumière nouvelle les relations entre les sources latines sur la première croisade.

Si l'on examine à présent dans quelle mesure les différentes sources ont été utilisées, on remarque que les Gesta Francorum et le nommé Pierre Tudebode (les chroniques «jumelles») constituent ensemble 57,2\% du texte, tandis que les passages dérivés d'un seul de ces textes n'en représentent que $4,5 \%$. On trouve aussi des passages où l'anonyme de l'HAI semble être passé d'un texte à l'autre, ligne après ligne, ou mieux mot après mot. 


\begin{tabular}{l|l|l} 
GF 2 & PT p. 33 & HAI \\
una pars Francorum & una pars in Ungarie & 4.2 Una pars Francorum \\
in Hungarie intrauit & intrauit regionem, scilicet & in Ungariae intrauit \\
regionem, scilicet Petrus & Petrus Heremita et dux & regionem, scilicet Petrus \\
Heremita et dux Gode- & Godefredus, et sapiens & Heremita et dux Gottifre- \\
fridus et Balduinus frater & Christi athleta Balduinus & dus et Baldoynus frater \\
eius et Balduinus comes de & frater eius. & eius, et comes Baldoynus \\
Monte. & & de Monte. \\
isti potentissimi milites & isti prudentissimi & 4.3 Isti prudentissimi \\
et alii plures quos ignoro & milites et alii quos ignoro, & milites et alii plures, quos \\
uenerunt per uiam quam & penitusque ductore careo, & ignoro et quorum nomina \\
iamdudum Karolus & uenerunt per uiam quam & penitus dictare careo, \\
Magnus, mirificus rex & iamdudum Carlomannus & uenerunt per uiam, quam \\
Francie, aptari fecit usque & mirificus rex Franciae & antiquitus Carolus, \\
Constantinopolim. & aptare fecit usque & mirificus rex Franciae, \\
Constantinopolim. & aptari fecit usque Costan- \\
tinopolim.
\end{tabular}

Mais il est tout à fait improbable que l'anonyme soit ainsi passé d'un texte à l'autre, comme nous le verrons plus avant.

Les sections originales de l'HAI (19,7\%) concernent surtout les contingents formés par les Normands d'Italie et les Provençaux. Or, il est possible qu'elles ne soient pas toutes « originales » : certains passages, par exemple, dérivent de la « rédaction $\mathrm{B}$ » des GF, soit la rédaction des GF non publiée par l'éditeur Bréhier. Voyons ici la manière dont est rapporté l'exploit de Robert fitzGerald, bras droit de Bohémond de Hauteville : 


\begin{tabular}{|c|c|c|c|}
\hline $\begin{array}{l}\text { GF rédac. A (= éd. } \\
\text { Bréhier) } 17\end{array}$ & GF rédac. $\mathrm{B}$ & PT p. 72 & HAI \\
\hline $\begin{array}{l}\text { Vade quam citius } \\
\text { potes, ut uir fortis }\end{array}$ & $\begin{array}{l}\text { Vade quam } \\
\text { citius potes ut uir } \\
\text { fortis et recordare } \\
\text { prudentium } \\
\text { antiquorumque } \\
\text { nostrorum fortium } \\
\text { parentum }\end{array}$ & $\begin{array}{l}\text { recordare pruden- } \\
\text { tium antiquorum } \\
\text { et nostrorum } \\
\text { fortium parentum, } \\
\text { quales fuerunt } \\
\text { et qualia bella } \\
\text { fecerunt }\end{array}$ & $\begin{array}{l}9.149 \text { «Uade quam } \\
\text { citius potes, et } \\
\text { recordare pruden- } \\
\text { tium antiquorum } \\
\text { et nostrorum } \\
\text { fortium <paren- } \\
\text { tum>, quales } \\
\text { fuerunt et qualia } \\
\text { bella fecerunt.» }\end{array}$ \\
\hline identique & identique & manquant & 9.150 \\
\hline identique & identique & manquant & 9.151 \\
\hline $\begin{array}{l}\text { fuit itaque ille, } \\
\text { undique signo } \\
\text { crucis munitus }\end{array}$ & $\begin{array}{l}\text { fuit itaque ille, } \\
\text { undique signo } \\
\text { crucis munitus }\end{array}$ & $\begin{array}{l}\text { iuit itaque ille, } \\
\text { undique signo } \\
\text { crucis armatus ut } \\
\text { fortissimus Christi } \\
\text { athleta, et sicut } \\
\text { sapiens et prudens }\end{array}$ & $\begin{array}{l}9.152 \text { iuit itaque } \\
\text { ille undique signo } \\
\text { crucis armatus, ut } \\
\text { fortissimus Christi } \\
\text { adleta, et sicut } \\
\text { sapiens et prudens }\end{array}$ \\
\hline identique & identique & manquant & 9.153 \\
\hline
\end{tabular}

Il apparait donc de manière évidente que toutes les parties «originales» de l'HAI ne le sont pas, et qu'elles dérivent d'une source écrite qui a existé mais que les chercheurs précédents ont oubliée. Il devient donc possible, voire probable, que les autres sections « originales» de l'HAI ne le soient pas non plus, mais dérivent de quelque source écrite actuellement perdue. Il est en effet très improbable que l'anonyme cassinien soit le seul en Europe, qui, distant des faits de 30 ou 40 ans, connaisse une série d'épisodes, de situations et de personnages absolument secondaires de la croisade et qui concernent en particulier le contingent provençal. On peut considérer, en revanche, que les épisodes qui se rapportent aux Normands d'Italie ont été connus du moine du Mont Cassin par des récits oraux : il est le seul à parler, par exemple, de la perte du cheval d'Hermann de Canne, un chevalier de Pouille, pendant une bataille. Voici la liste des épisodes «provençaux» dont l'HAI est l'unique source :

- 6.33 deux membres de la première ambassade croisée au Caire portent des noms qui semblent évidemment provençaux : Bertrand de Scabrita et Pierre de Poitiers;

- 9.103 l'HAI est la seule chronique qui parle de la mort et de la sépulture du chevalier provençal Pierre Raymond de Hautpoul;

- 9.227 dans une liste de cavaliers provençaux qui restent à la garde d'une porte d'Antioche (de GF 11/PT p. 78), l'HAI seule mentionne Gérard Malafaida; 
- 9.228 et 15.57 générosité de Raymond de Saint-Gilles;

- 9.229 parmi les morts d'une attaque turque à la Mahomerie, l'HAI seule ajoute Bernard de Pardilo;

- 11.26 protection divine pour Raymond de Saint-Gilles;

- 14.3 l'HAI est l'unique source pour la présence de Raymond de Turenne et de Geoffroi de Lastours à l'expédition de Raymond Pilet contre Tel Mannas;

- 14.15-17 l'HAI est l'unique source rappelant une bagarre victorieuse de Raymond Pilet et Raymond de Turenne le jour de l'Assomption (GF 30/PT p. 115);

- 15.54 l'HAI est l'unique source pour le nom du chef du groupe de Provençaux envoyés par le comte de Saint-Gilles pour embellir sa résidence d'Antioche : Guillaume Ermingareo;

- 19.5-10 il n'existe pas d'autre source pour l'affrontement entre Raymond de Saint-Gilles et Geoffroi de Bouillon sur la question du serment de Raymond à Geoffroi.

Ainsi, comme Jay Rubenstein l'avait déjà supposé, l'HAI n'emprunte pas aux GF ni à PT séparément, mais à une source commune (Common Source - CS) aujourd'hui perdue, qui leur aurait servi de texte de base et qu'il appelle « Jerusalem History » ${ }^{5}$. À partir de cette œuvre ont été composés les GF et PT sous la forme que nous leur connaissons aujourd'hui (archétype statique). En revanche, Jean Flori pense à une Common Source qui aurait «évolué » dans le temps et dans l'espace, et serait devenue à la fois GF et PT (archétype dynamique) ${ }^{6}$.

Mais qu'il ait été statique ou dynamique, il faut en tous cas postuler l'existence d'un texte unique utilisé par l'auteur de l'HAI, qui n'a donc lu ni les GF ni PT séparément : penser à un collage «mot par mot » entre les deux textes est simplement absurde, et n'explique pas la présence des notices originales.

\section{"La mort de Roger de Barneville» : vers un nouveau stemma des sources de la première croisade}

Examinons maintenant un cas spécifique:l'épisode de la mort de Roger de Barneville (HAI 10.19-23) 7 . Le 4 juin 1098, les Croisés sont assiégés dans Antioche. À l'arrivée des avant-gardes de l'armée de Kerbogha, le chevalier normand Roger de Barneville sort avec un petit nombre pour inspecter les mouvements des Turcs. Mais les forces ennemies attaquent les Croisés, qui cherchent à se sauver à l'intérieur des murs. Le cheval de Roger trébuche dans les marais du fleuve, si bien que le chevalier, aussitôt encerclé par les Turcs, se protège derrière son cheval et sort son épée. Malgré sa bravoure, ses ennemis le transpercent avec

5. RubENSTEIN, 2005.

6. FLORI, 2007, p. 717 et $739-745$

7. Nous indiquons entre crochets $<>$ les intégrations faites sur la base du manuscrit parisien. 
leurs longs épieux (longis cannis). Dans la douleur la plus grande, les Croisés récupèrent le corps et lui donnent une sépulture dans la cathédrale d'Antioche.

10.18 Tertia autem die postquam ciuitatem <intrauimus, precursores illorum ante civitatem > precurrerunt. 10.19 Roggerius igitur de Bernauilla contra <eos $>$ exiuit solummodo cum uiginti militibus : appropinquantes autem Turci ceperunt continuo preliari cum eis. 10.20 Forte equus, in quo sedebat, in quadam <retentus > palude, infixus est <in ea $>$ et cecidit : erectus autem statim est <ille $>$ super suos pedes et, euaginato ense, mirabiliter defendebat se de Turcorum inuasione. 10.21 Tunc accedentes Arabes cum longis cannis uulnerauerunt <eum ad mortem apprehenderuntque eum et detruncauerunt $>$ caput eius. 10.22 Nos autem subleuauimus corpus eius a terra et deportauimus in ciuitatem ad Sancti Petri ecclesiam; ibique honestissime sepultus est, cuius anima exultat cum angelis. 10.23 Unde maxima tristitia atque dolor fuit in tota Christi militia, quia acerrimus ille erat atque bellicosus miles, ipseque multos militares actus faciebat.

On s'aperçoit que le texte du manuscrit cassinien 300, apparemment autosuffisant, est en fait entaché d'une erreur si on le confronte au texte du manuscrit parisien : l'erreur vient de deux évidents « sauts du-même-au-même » effectués par le scribe :

- HAI 10.18 : C om. intrauimus, precursores illorum ante ciuitatem (le mot précédant cette phrase est ciuitatem);

- HAI 10.21: Com. eum ad mortem apprehenderuntque eum et detruncauerunt (le mot précédant cette phrase est uulnerauerunt).

Cet épisode, connu seulement dans la version du manuscrit cassinien $(\mathrm{RHC})^{8}$, n'avait jamais été analysé. Tandis qu'il est absent dans GF/PT, il est présent dans les textes dérivés des GF (RM, notamment) ou les textes autonomes (Albert d'Aix : AA), mais les versions de l'épisode sont différentes. Il y a, il faut le souligner ici, un autre passage où l'HAI donne des notices absentes des sources écrites qu'il a compilées habituellement. En HAI 9.305 : le premier Croisé à se hisser sur les murs de Jérusalem fut Paganus Longobardus. Cette notice, absente des chroniques jumelles (GF-PT), ne peut pas être une information personnelle de l'anonyme de l'HAI, car elle est présente dans le texte de Baudri de Dol 2.20, "quidam Longobardus nomine Paganus".

Voici une représentation synoptique des éléments narratifs présents dans chaque source :

8. Voir Historia de via Hierusolymis, 1866, p. 198.

URL : http://tabularia.revues.org/288o | DOI : 10.400o/tabularia.2880 


\begin{tabular}{|c|c|c|c|c|}
\hline & RA p. 66 & RM 6.8 & $\mathrm{AA}_{4.27}$ & HAI 10.19-23 \\
\hline $\begin{array}{l}\text { conditions de } \\
\text { la bagarre }\end{array}$ & $\begin{array}{l}\text { poursuite } \\
\text { turque }\end{array}$ & $\begin{array}{l}\text { fuite depuis } \\
\text { le lieu de } \\
\text { l'embuscade } \\
\text { turque }\end{array}$ & $\begin{array}{l}\text { fuite depuis le } \\
\text { lieu de l'embus- } \\
\text { cade turque }\end{array}$ & $\begin{array}{l}\text { fuite depuis le } \\
\text { lieu de l'embus- } \\
\text { cade turque }\end{array}$ \\
\hline $\begin{array}{l}\text { équipe des } \\
\text { Croisés }\end{array}$ & & 3 cavaliers & 15 cavaliers & 20 cavaliers \\
\hline difficultés & capture & chute de cheval & lenteur du cheval & $\begin{array}{l}\text { chute du cheval } \\
\text { (marais) }\end{array}$ \\
\hline blessure & & & $\begin{array}{l}\text { un Turc à cheval } \\
\text { blesse Roger avec } \\
\text { une flèche }\end{array}$ & $\begin{array}{l}\text { des Arabes } \\
\text { blessent Roger } \\
\text { avec des épieux } \\
\text { (cannis) }\end{array}$ \\
\hline $\begin{array}{l}\text { localisation de } \\
\text { la blessure }\end{array}$ & & $\begin{array}{l}\text { massacre du } \\
\text { corps }\end{array}$ & foie et poumon & \\
\hline décapitation & oui & oui & oui & oui \\
\hline sépulture & & & dans l'église & $\begin{array}{l}\text { dans la cathé- } \\
\text { drale }\end{array}$ \\
\hline $\begin{array}{l}\text { douleur des } \\
\text { Croisés }\end{array}$ & oui & oui & oui & oui \\
\hline
\end{tabular}

AA présente une version des faits assez fantaisiste (il est le seul qui ne parle pas de la chute du cheval).

HAI : la version la plus simple et en même temps la plus détaillée est celle de l'HAI. L'anonyme évoque le détail réaliste des marais alimentés par le fleuve Oronte sous les murs d'Antioche, décrit le cavalier qui, après la chute de son cheval, se redresse, l'épée à la main, et les Arabes qui interviennent avec leurs longs épieux (longis cannis) pour le frapper de loin.

Mais le plus intéressant vient du fait que les matériaux linguistiques de ce récit sont typiques du style des «sources jumelles» (GF/PT), comme en témoigne la lecture comparée de ces sources :

HAI 10.19 Roggerius igitur de Bernauilla contra $<$ eos $>$ exiuit solummodo cum uiginti militibus : appropinquantes autem Turci ceperunt continuo preliari cum eis;

GF 30/PT p. 116 exierunt ergo barbari contra illos ad bellum;

GF 11/PT p. 61 mox preparauit se solummodo cum militibus quatinus illos undique expugnaret;

PT p. 62 tunc perrexit cum suis honestissimis militibus et seruientibus;

PT p. 58 uenit Tharso solummodo cum suis militibus;

GF 8/PT p. 50 non potuerunt preliari cum illis. 
HAI 10.20 Forte equus, in quo sedebat, in quadam <retentus $>$ palude, infixus est <in ea $>$ et cecidit : erectus autem statim est <ille > super suos pedes et, euaginato ense, mirabiliter defendebat se de Turcorum inuasione;

GF 26/PT p. 102 et tercius per totum diem viriliter defendebat se de Turcorum invasione;

GF 34/PT p. 128 mirabiliter munierant castrum illud et defendebant se fortiter.

HAI 10.21 Tunc accedentes Arabes cum longis cannis uulnerauerunt <eum ad mortem apprehenderuntque eum et detruncauerunt $>$ caput eius ;

GF 30 apprehenderunt igitur omnes illius loci colonos et qui christianitatem recipere noluerunt, occiderunt;

GF 39 eumque uulnerauit usque ad mortem;

GF 17/PT p. 42 et occiderunt et apprehenderunt multos ex eis;

GF 20/PT p. 87 apprehenderunt eum, truncaueruntque caput illius.

HAI 10.22 Nos autem subleuauimus corpus eius a terra et deportauimus in ciuitatem ad Sancti Petri ecclesiam; ibique honestissime sepultus est, cuius anima exultat cum angelis;

GF 18/PT p. 77 deportauerunt cesa capita ad tentoria;

GF 18/PT p. 77 sepelierunt ad Machumariam;

PT p. 80 animam cuius angeli confestim suscipientes ante conspectum Dei pro cuius amore martyrium suscepit, gaudentes et psallentes detulerunt;

PT p. 116 cuius sanctissima anima felix exultat cum angelis.

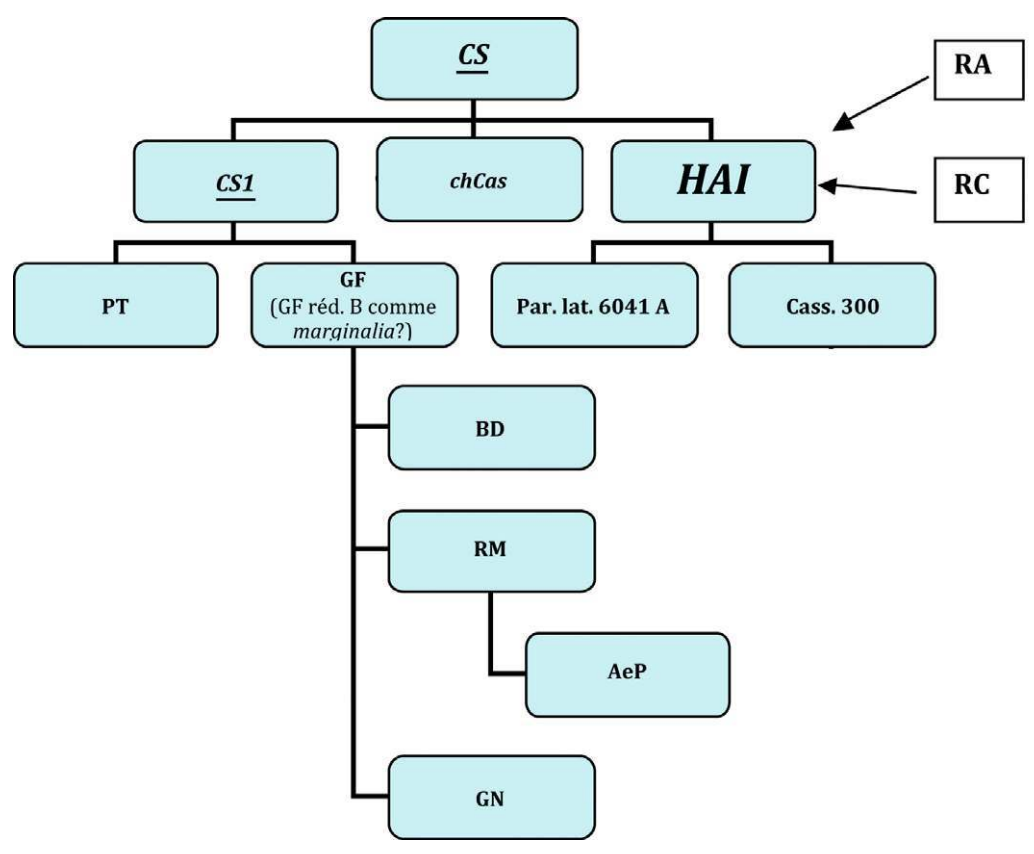

Fig. 2 : Stemma 
HAI 10.23 Unde maxima tristitia atque dolor fuit in tota Christi militia, quia acerrimus ille erat atque bellicosus miles, ipseque multos militares actus faciebat;

PT p. 62 tristes, plaudebant manibus pre nimia tristicia atque dolore;

GF 30/PT p. 117 unde magna angustia et tribulatio immensusque dolor fuit in tota Christi militia;

GF 7 et fuit maxima ubertas in tota Christi militia;

GF 34 unde ualde fuit refecta tota Christi militia;

GF 36 unde nimis ditata est omnis Christi militia.

On retrouve la même identité du tissu stylistique entre l'HAI et les chroniques jumelles dans un autre épisode « original » de l'HAI : il s'agit de l'envoi de la première ambassade croisée au Caire (HAI 6.32-36).

\section{Conclusion}

Ainsi, l'épisode de Roger de Barneville est un cas particulier de la section de l'HAI considérée jusqu'ici comme originale, mais qui en fait était présente dans une source écrite à présent perdue. Cette source - la Common Source de Jay Rubenstein et Jean Flori - avait les caractéristiques stylistiques des chroniques jumelles. La seule explication possible est que l'anonyme de l'HAI (comme les auteurs des autres sources dérivées apparemment des $\mathrm{GF}^{9}$, qui présentent l'épisode de la mort de Roger de Barneville) n'a pas lu le texte de «nos » GF + PT, mais le texte dont ces deux «œuvres» dérivent elles-mêmes ${ }^{10}$.

La présentation stemmatique du développement textuel d'une partie du corpus des sources sur la première croisade peut être la suivante (le soulignement met en évidence les états de rédaction que nous proposons comme hypothèse). Il faudrait maintenant joindre à ce stemma le texte nommé Peregrinatio Antiochie per Vrbanum papam facta, récemment étudié par Samu Niskanen ${ }^{11}$ (voir fig. 2).

. RM, RA, AA

10. Russo, 2014.

11. Niskanen, 2012. Cet article pèche malheureusement par une excessive assurance sur des problèmes qui sont plus complexes que l'auteur semble le penser. En outre, l'auteur n'est pas du tout sûr de la place précise de cette Peregrinatio dans le stemma des chroniques latines sur la croisade (p. 295-296). 


\begin{tabular}{|c|c|}
\hline $\begin{array}{l}\text { CS (Common } \\
\text { Source) }\end{array}$ & $\begin{array}{l}\text { - écrit en Terre Sainte - ante } 1102 \\
\text { - coïncide avec A1 de Flori - coïncide avec «Jerusalem } \\
\text { History» de Rubenstein } \\
\text { - riche de variantes dans les marges, entre les lignes, } \\
\text { documents, gloses, feuillets ajoutés, etc. } \\
\text { - idéologie : pas de position politique marquée en faveur des } \\
\text { Normands ou des Provençaux - présence importante des } \\
\text { Provençaux } \\
\text { - contenu : passages des GF - passages de PT - toutes les } \\
\text { adjonctions de la rédaction B des GF - quelques éléments } \\
\text { «originaux» de l'HAI (Roger de Barneville, Paganus } \\
\text { Longobardus, négociations de Tancrède pour le château } \\
\text { d'Antioche, noms de chevaliers provençaux, etc.). }\end{array}$ \\
\hline $\mathrm{CS}_{1}$ & $\begin{array}{l}\text { - écrit } c a 1104 \\
\text { - coïncide avec A2 de Flori } \\
\text { - contenu : absence des parties « originales » de l'HAI. }\end{array}$ \\
\hline $\begin{array}{l}\text { GF (avec les adjonc- } \\
\text { tions de la rédaction } \\
\text { B des GF (dans les } \\
\text { marges?) }\end{array}$ & $\begin{array}{l}\text { - écrit ca } 1106 \\
\text { - coïncide avec A3 de Flori } \\
\text { - contenu : esprit antibyzantin et antiprovençal } \\
\text { - contenu : toutes les adjonctions de la rédaction B des GF } \\
\text { (dans les marges?). }\end{array}$ \\
\hline
\end{tabular}

URL : http://tabularia.revues.org/2880 | DOI : 10.400o/tabularia.2880 


\section{Bibliographie}

\section{Sources}

Albert D'Aix, Albert of Aachen Historia Ierosolimitana : history of the journey to Jerusalem, éd. Susan B. Edgington, Oxford, Clarendon Press (Oxford Medieval texts), 2007.

Anonymi Gesta Francorum et aliorum Hierosolimitanorum, éd. Heinrich HAGENMEYer, Heidelberg, C. Winter, 1890.

BAUDRI DE DoL, «Baldrici episcopi Dolensis Historia Jerosolimitana », in Recueil des historiens des croisades. Historiens occidentaux, t. IV, Paris, Imprimerie nationale, 1879, p. 1-112 (ark :/12148/bpt6k515745).

«Chronica monasterii Casinensis", in Monumenta Germaniae historica. Scriptores, éd. Hartmut Hoffmann, t. XXXIV, Hannovre, Hahn, 1980.

Foucher de Chartres, Fulcherii Carnotensis Historia Hierosolymitana (1095-1127), éd. Heinrich Hagenmeyer, Heidelberg, C. Winter, 1913.

Gilles de Paris, The Historia vie Hierosolimitane of Gilo of Paris, and a second, anonymous author, éd. Christopher Wallace Grocock, Elizabeth Siberry, Oxford, New York, Oxford University (Oxford Medieval Texts), 1997, 1 vol.

Guibert de Nogent, Dei Gesta per Francos et cinq autres textes, éd. Robert Burchard Constantijn Huygens, Turnhout, Brepols (Corpus Christianorum. Continuatio Mediaevalis, $\mathrm{n}^{\circ} 127$ A), 1996.

Histoire anonyme de la première croisade, éd. Louis BréHIER, Paris, Honoré Champion (Les classiques de l'histoire de France au Moyen Âge, vol. 4), 1924 (édition de référence pour le texte). Autres éditions : Gesta Francorum et aliorum Hierosolimitanorum, éd. Rosalind Hill, Londres, Édimbourg, Thomas Nelson \& Sons, 1962; Le Gesta dei Franchi e degli altri pellegrini gerosolimitani, éd. Luigi Russo, Alessandria, Éditions dell'Orso (Il cavaliere del leone, vol. 6), 2003.

"Historia de via Hierusolymis", in Recueil des historiens des Croisades. Historiens occidentaux, Académie royale des Inscriptions et des Belles Lettres, t. III, Paris, 1866, p. 169-229 (ark :/12148/bpt6k51573t).

Hystoria de via et recuperatione Antiochiae atque Ierusolymarum (olim Tudebodus imitatus et continuatus), éd. Edoardo D'Angelo, Florence, Sismel - édition del Galluzzo (Edizione Nazionale dei testi mediolatini, $\mathrm{n}^{\circ} 23$ ), 2009, 1 vol.

Pierre Tudebode, Historia de Hierosolymitano itinere, éd. John Hugh Hill, Laurita Littleton HiLl, Paris, librairie orientaliste Paul Geuthner (Documents relatifs à l'histoire des croisades, $\left.\mathrm{n}^{\circ} 12\right), 1977$.

Raoul de Caen, "Gesta Tancredi in expeditione Hierosalymitana/auctore Radulfo Cadomensi ", in Recueil des historiens des croisades. Historiens occidentaux, Imprimerie nationale, Académie royale des Inscriptions et des Belles Lettres, t. III, Paris, 1866, p. 587-716 (ark :/12148/bpt6k51573t).

Raoul de Caen, Radulphi Cadomensis Tancredus, éd. Edoardo D’Angelo, Turnhout, Brepols (Corpus Christianorum. Continuatio Mediaevalis, $\mathrm{n}^{\circ} 231$ ), 2011.

Raymond D'Aguilers, Le «Liber » de Raymond d'Aguilers, éd. John Hugh Hill, Laurita Littleton HiLl, Paris, Geuthner (Documents relatifs à l'histoire des croisades, $\mathrm{n}^{\circ}$ 9), 1969. 
Robert le Moine, "Roberti Monachi Historia Iherosolimitana", in Recueil des historiens des Croisades. Historiens occidentaux, t. III, Paris, Imprimerie impériale, Académie royale des Inscriptions et des Belles Lettres, 1866, p. 719-882 (ark :/12148/ bpt6k51573t).

\section{Études}

Flori, Jean, «De l'Anonyme normand à Tudebode et aux Gesta Francorum. L'impact de la propagande de Bohémond sur la critique textuelle des sources de la première croisade", Revue d'Histoire Ecclésiastique, 102, 2007, p. 717-746.

France, John, «The Use of the Anonymous Gesta Francorum in the early twelfthcentury sources for the First Crusade», in From Clermont to Jerusalem : The Crusades and Crusader Society 1095-1500, Alan V. Murray (éd.), Turnhout, Brepols, 1998, p. 29-42.

Krey, August Charles, "A Neglected Passage in the Gesta Francorum and its Brearing on the Literature of the First Crusade ", in The Crusades and Other Historical Essays presented to D.C. Munro, Louis John Paetow (éd.), New York, F.S. Crofts, 1928, p. 57-78.

Rubenstein, Jay, "What is the Gesta Francorum, and who was Peter Tudebode?», Revue Mabillon, 16, 2005, p. 179-204.

Russo, Luigi, «Le fonti della "prima crociata" ", in Mediterraneo medievale. Cristiani, musulmani ed eretici tra Europa e Oltremare, (secoli IX-XIII), Marco MeschINI (éd.), Milano, Vita e pensiero, 2001, p. 51-65.

Russo, Luigi, "The Monte Cassino Tradition of the First Crusade : from the Chronica Monasterii Casinensis to the Hystoria de via et recuperatione Antiochiae atque Ierusolymarum ", in Writing the Early Crusades: Texts, Transmission and Memory, Marcus Bull et Damien KeMPf (dir.), Woodbridge, Boydell Press, 2014, p. 53-62.

Niskanen, Samu, «The Origins of the Gesta Francorum and two Related Texts : their Textual and Literary Character», Sacris Erudiri, 51, 2012, p. 287-316.

Wolf, Kenneth Baxter, «Crusade and Narrative : Bohemund and the Gesta Fancorum », Journal of Medieval History, 17, 1991, p. 207-216. 